\title{
Sentinel node biopsy for melanoma: unnecessary treatment?
}

In the course of researching this Feature on sentinel lymph node (SLN) biopsy (BMJ 2013;346:e8645, doi:10.1136/bmj.e8645) we were not clear with one of the interviewees who kindly spoke to our reporter how any of the interview might be used in the final article. In addition, some of the quotes attributed to Marc Moncrieff misrepresented his views. We would like to make it clear that in the transcript of the interview Mr Moncrieff makes positive comments about the practice of SLN biopsy and we should have included a proportion of them. This would have led to a fairer report.

Mr Moncrieff does not believe that the use of SLN biopsy leads to overtreatment, but that the majority of patients who have a lymphadenectomy after SLN biopsy have no evidence of further disease. Accordingly, the MSLT-II trial hopes to identify those patients who may not benefit from a completion lymphadenectomy (and thus avoid any further surgery) by the use of ultrasound follow-up alone.

In the Feature, Mr Moncrieff says: "Eighty-eight per cent of patients don't have further disease in their nodal basin." He would like to make it clear that this $88 \%$ figure only relates to patients studied at the Norfolk and Norwich University Hospitals Foundation Trust.

We apologise to Marc Moncrieff for misrepresenting his views.

Cite this as: BMJ 2013;346:f272

๑ BMJ Publishing Group Ltd 2013 